\title{
Natural convection on a vertical cylinder embedded in a thermally stratified high-porosity medium
}

\author{
H.S. Takhar ${ }^{\text {a,* }}$, A.J. Chamkha ${ }^{\text {b }}$, G. Nath ${ }^{\text {c }}$ \\ a Department of Engineering, Manchester Metropolitan University, Manchester, M1 5GD, UK \\ b Department of Mechanical Engineering, Kuwait University, P.O. Box 5969, Safat, Kuwait \\ ${ }^{\mathrm{c}}$ Department of Mathematics, Indian Institute of Science, Bangalore-560012, India
}

Received 26 May 2000; accepted 12 March 2001

\begin{abstract}
The laminar natural convection boundary layer flow on an isothermal vertical thin cylinder embedded in a thermally stratified high porosity medium has been studied. The non-Darcy effects including convective, boundary and inertial effects are included in this analysis. The non-linear coupled partial differential equations governing the flow are solved by using an implicit finite-difference scheme. For small and moderate values of the axial distance, these equations are also solved by a perturbation technique in combination with the Shanks transformation. It is observed that for certain values of the ambient stratification parameter, the skin friction vanishes and the direction of the heat transfer changes. The skin friction and heat transfer decrease with increasing ambient stratification, curvature and inertia parameters, but they increase with the permeability parameter. @ 2002 Éditions scientifiques et médicales Elsevier SAS. All rights reserved.
\end{abstract}

Keywords: Stratified natural convection; Flow through porous media

\section{Introduction}

Heat transfer by natural convection frequently occurs in many physical problems and engineering applications such as geothermal systems, heat exchangers, chemical catalytic reactors, fiber and granular insulation, packed beds, petroleum reservoirs and nuclear waste repositories. Tien and Vafai [1] have presented an excellent review of natural convection flow in porous media and have stressed the importance of the non-Darcy effects such as the inertia and boundary effects. Chen et al. [2] have investigated the non-Darcy effects on the natural convection boundary layer flow on an isothermal vertical flat surface placed in a high-porosity medium. Certain porous materials, such as foam metals and fibrous media have a high porosity $(0.9 \leqslant$ $\varepsilon \leqslant 0.95)$. Thermal stratification occurs in cooling ponds, lakes, solar ponds and in the atmosphere. If the vertical surface is a part of an enclosure, the ambient temperature of the fluid will be stratified. For stable thermal stratification, the temperature in the ambient fluid increases with height. Singh and Tewari [3] have studied the non-Darcy natural

\footnotetext{
* Correspondence and reprints.

E-mail address: h.s.takhar@mmu.ac.uk (H.S. Takhar).
}

convection boundary layer flow over a vertical isothermal plate immersed in a thermally stratified fluid saturated porous medium. Chen and Lin [4] have investigated the above problem for a highly porous medium. Chamkha [5] has extended the analysis of Chen and Lin [4] to include the effect of the magnetic field.

Natural convection flow over a vertical flat surface in a thermally stratified non-porous medium has been studied by Eichhorn [6], Chen and Eichhorn [7] and Venkatachala and Nath [8], who used the series solution method, the local nonsimilarity method and the finite difference method, respectively, for the solution of the governing partial differential equations. Kulkarni et al. [9] have obtained a similarity solution for the natural convection flow over a heated vertical plate in a thermally stratified fluid. Angirasa and Srinivasan [10] have presented a numerical study of the natural convection flow on a vertical surface due to the combined effect of buoyancy forces caused by the heat and mass diffusion in a thermally stratified medium. The natural convection flow over a horizontal cylinder and a sphere immerged in a thermally stratified fluid was investigated by Chen and Eichhorn [11].

In the present study, the natural convection flow on a vertical isothermal thin cylinder embedded in a thermally strat- 


\begin{tabular}{|c|c|c|}
\hline \multicolumn{3}{|c|}{ Nomenclature } \\
\hline$A$ & gradient of ambient temperature. & $\ldots \ldots \ldots \mathrm{K}$ \\
\hline$c_{\mathrm{f}}$ & specific heat of the fluid .......... & $\mathrm{J} \cdot \mathrm{kg}^{-1} \cdot \mathrm{K}^{-1}$ \\
\hline C & dimensionless inertia parameter & \\
\hline$C^{*}$ & dimensionless inertia parameter & \\
\hline$C_{\mathrm{f} x}$ & local skin friction coefficient & \\
\hline$e$ & operator & \\
\hline$f$ & dimensionless stream function & \\
\hline$g$ & acceleration due to gravity.... & $\mathrm{m} \cdot \mathrm{s}^{-2}$ \\
\hline$G r$ & Grashof number & \\
\hline$G r_{x}$ & local Grashof number & \\
\hline$k_{e}$ & $\begin{array}{l}\text { effective thermal conductivity } \\
\text { of the porous medium } . . . . . .\end{array}$ & $\mathrm{W} \cdot \mathrm{m}^{-2} \cdot \mathrm{K}^{-1}$ \\
\hline K & dimensionless permeability & \\
\hline$K^{*}$ & dimensionless permeability & \\
\hline$L_{j}$ & partial sum & \\
\hline$N u_{x}$ & local Nusselt number & \\
\hline $\operatorname{Pr}$ & Prandtl number & \\
\hline$r, x$ & radial and axial coordinates.... & $\ldots \ldots \ldots m$ \\
\hline$r_{0}$ & radius of the cylinder $\ldots \ldots \ldots \ldots$ & $\ldots \ldots \ldots m$ \\
\hline$S$ & $\begin{array}{l}\text { dimensionless ambient thermal str } \\
\text { parameter }\end{array}$ & tification \\
\hline$T$ & Temperature ................. & K \\
\hline
\end{tabular}

ified high porosity medium has been considered. The nonlinear coupled partial differential equations governing the flow have been solved numerically using an implicit finitedifference scheme similar to that of Blottner [12]. For small and moderate values of the axial distance, these equations have also been solved by using a perturbation technique [13] along with the Shanks transformation. Natural convection flow over a vertical thin cylinder without thermal stratification and a porous medium has been investigated by a number of research workers [13-17]. The results in the absence of the stratification and porous medium are compared with those of Aziz and $\mathrm{Na}$ [13], Cebeci [16] and Minkowycz and Sparrow [17].

\section{Analysis}

The physical model of the problem and the coordinate system are given in Fig. 1. Consider a vertical thin circular cylinder of radius $r_{0}$ maintained at a uniform temperature $T_{\mathrm{w}}$ situated in a stable stratified ambient fluid at temperature $T_{\infty}(x)$. The cylinder is embedded in a high-porosity medium. The radial coordinate $r$ is measured from the axis of the cylinder and the axial coordinate $x$ is measured vertically upward such that $x=0$ corresponds to the leading edge where the boundary layer thickness is zero. The conventional assumptions of the laminar boundary layer theory are applied and the Boussinesq approximation is invoked.
$T_{0} \quad$ ambient temperature at $x=0 \ldots \ldots \ldots \ldots$ K

$T_{\infty} \quad$ ambient temperature $\ldots \ldots \ldots \ldots \ldots \ldots \ldots \mathrm{K}$

$u, v \quad$ velocity components along the radial

and axial directions . . . . . . . . . . $\mathrm{m} \cdot \mathrm{s}^{-1}$

$U^{*} \quad$ reference velocity................ $\mathrm{m} \cdot \mathrm{s}^{-1}$

$\alpha_{\mathrm{e}} \quad$ effective thermal diffusivity

of the medium .............. $\mathrm{m}^{2} \cdot \mathrm{s}^{-1}$

$\beta \quad$ volumetric coefficient of thermal

expansion $\ldots \ldots \ldots \ldots \ldots \ldots \ldots \ldots, \mathrm{K}^{-1}$

$\varepsilon \quad$ porosity of the medium

$\eta, \xi \quad$ transformed coordinates

$\theta$ dimensionless temperature

$\mu \quad$ coefficient of viscosity ........ kg.m $\mathrm{m}^{-1} \cdot \mathrm{s}^{-1}$

$v \quad$ kinematic viscosity $\ldots \ldots \ldots \ldots \ldots \mathrm{m}^{2} \cdot \mathrm{s}^{-1}$

$\rho_{\mathrm{f}} \quad$ density of the fluid $\ldots \ldots \ldots \ldots \ldots . . \ldots \ldots . \mathrm{kg}^{-3}$

$\Psi \quad$ dimensional stream function ..... $\mathrm{kg} \cdot \mathrm{m}^{-1} \cdot \mathrm{s}^{-1}$

Subscripts

$w, \infty$ conditions at the wall and in the ambient fluid

Superscript

prime denotes derivative with respect to $\eta$

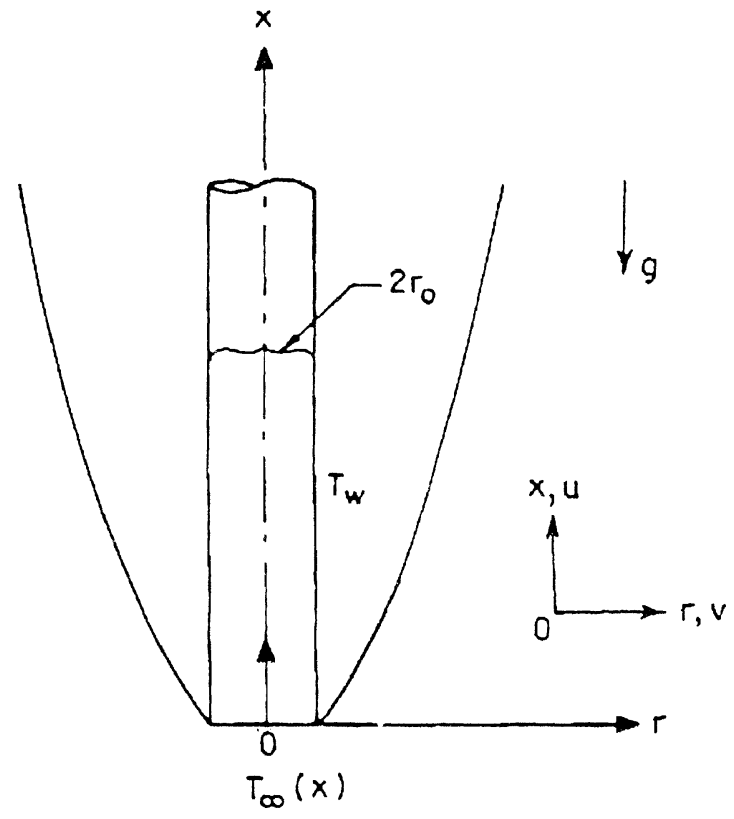

Fig. 1. Physical model and coordinate system.

Under the foregoing assumptions, the governing equations can be expressed as $[4,7,13,17]$

$$
\begin{aligned}
& \frac{\partial}{\partial x}(r u)+\frac{\partial}{\partial r}(r v)=0 \\
& \varepsilon^{-2}\left(u \frac{\partial u}{\partial x}+v \frac{\partial u}{\partial r}\right)=g \beta\left(T-T_{\infty}(x)\right)
\end{aligned}
$$


$+\varepsilon^{-1} \frac{v}{r} \frac{\partial}{\partial r}\left(r \frac{\partial u}{\partial r}\right)-\left(\frac{v}{K^{*}}\right) u-C^{*} u^{2}$

$u \frac{\partial T}{\partial x}+v \frac{\partial T}{\partial r}=\frac{\alpha_{\mathrm{e}}}{r} \frac{\partial}{\partial r}\left(r \frac{\partial T}{\partial r}\right)$.

The boundary conditions are

$$
\begin{array}{ll}
u=v=0, \quad T=T_{\mathrm{w}} & \text { at } r=r_{0}, x \geqslant 0 \\
u \rightarrow 0, \quad T \rightarrow T_{\infty}(x) & \text { as } r \rightarrow \infty, x \geqslant 0 \\
u=0, \quad T=T_{\infty}(x) & \text { at } x=0, r>r_{0} .
\end{array}
$$

Here $r$ and $x$ are the radial and axial coordinates, respectively; $u$ and $v$ are the velocity components along the $r$ and $x$ directions, respectively; $T$ is the temperature; $\varepsilon$ is the porosity; $g$ is the acceleration due to the gravity; $\beta$ is the volumetric coefficient of thermal expansion; $K^{*}$ is the permeability; $C^{*}$ is the inertia coefficient; $\alpha_{\mathrm{e}}\left(=k_{\mathrm{e}} / \rho_{\mathrm{f}} c_{\mathrm{f}}\right)$ is the effective thermal diffusivity of the medium; $k_{\mathrm{e}}$ is the effective thermal conductivity of the saturated porous medium; $\rho_{\mathrm{f}}$ is the density of the fluid; $C_{\mathrm{f}}$ is the specific heat of the fluid; $r_{0}$ is the radius of the cylinder; and the subscripts $w$ and $\infty$ denote conditions at the wall and in the ambient fluid, respectively.

In order to make Eqs. (1)-(3) dimensionless, we apply the following transformations;

$$
\begin{aligned}
& T_{\infty}(x)=T_{0}+a x, \quad a=\mathrm{d} T_{\infty} / \mathrm{d} x>0, \\
& \eta=\left[g \beta\left(T_{\mathrm{w}}-T_{0}\right) / 4 v^{2}\right]^{1 / 4}\left(r^{2}-r_{0}^{2}\right) /\left(2 r_{0} x^{1 / 4}\right) \\
& \xi=2\left(x / r_{0}^{2}\right)^{1 / 4} /\left[g \beta\left(T_{\mathrm{w}}-T_{0}\right) r_{0}^{2} / 4 v^{2}\right]^{1 / 4} \\
& \psi(x, r)=4 v r_{0} x^{3 / 4}\left[g \beta\left(T_{\mathrm{w}}-T_{0}\right) r_{0}^{2} / 4 v^{2}\right]^{1 / 4} f(\xi, \eta) \\
& r u=\partial \psi / \partial r, \quad r v=-\partial \Psi / \partial x \\
& T(x, r)-T_{\infty}(x)=\left(T_{\mathrm{w}}-T_{0}\right) \theta(\xi, \eta) \\
& K=K^{*} / r_{0}^{2}, \quad C=4 C^{*} r_{0} G r \\
& G r=g \beta\left(T_{\mathrm{w}}-T_{0}\right) r_{0}^{3} / 4 v^{2} \\
& G r_{x}=g \beta\left(T_{\mathrm{w}}-T_{0}\right) x^{3} / 4 v^{2} \\
& S=a G r r_{0} /\left(T_{\mathrm{w}}-T_{0}\right) \\
& P r=v / \alpha_{\mathrm{e}}
\end{aligned}
$$

to Eqs. (1)-(3) and we find that Eq. (1) is identically satisfied and Eqs. (2) and (3) reduce to;

$$
\begin{aligned}
& \varepsilon^{-1}(1+\xi \eta) f^{\prime \prime \prime}+\varepsilon^{-1} \xi f^{\prime \prime}+\varepsilon^{-2}\left(3 f f^{\prime \prime}-f^{\prime 2}\right) \\
&+\theta-K^{-1}(\xi / 2)^{2} f^{\prime}-C(\xi / 2)^{4} f^{\prime 2} \\
&= \varepsilon^{-2} \xi\left(f^{\prime} \partial f^{\prime} / \partial \xi-f^{\prime \prime} \partial f / \partial \xi\right) \\
&(1+\xi \eta) \theta^{\prime \prime}+\xi \theta^{\prime}+3 \operatorname{Pr} f \theta^{\prime}-4 \operatorname{Pr}(\xi / 2)^{4} S f^{\prime} \\
&=\operatorname{Pr} \xi\left(f^{\prime} \partial \theta / \partial \xi-\theta^{\prime} \partial f / \partial \xi\right) .
\end{aligned}
$$

The boundary conditions (4) can be rewritten as

$$
\begin{aligned}
& f(\xi, 0)=f^{\prime}(\xi, 0)=0 \\
& \theta(\xi, 0)=1-S(\xi / 2)^{4} \\
& f^{\prime}(\xi, \infty)=\theta(\xi, \infty)=0 .
\end{aligned}
$$

Here $\xi$ and $\eta$ are the transformed coordinates; $\psi$ and $f$ are the dimensional and dimensionless stream functions, respectively; $K$ is the dimensionless permeability; $C$ is the dimensionless inertia coefficient; $\theta$ is the dimensionless temperature; $G r$ is the Grashof number, $G r_{x}$ is the local Grashof number; $P r$ is the Prandtl number; $S$ is the dimensionless ambient thermal stratification parameter; $T_{0}$ is the value of the ambient temperature $T_{\infty}$, at $x=0$; a is the slope of the ambient temperature and $a>0$ for a stable stratified fluid, and prime denotes derivative with respect to $\eta$.

It may be noted that Eqs. (6)-(8) for $S=0$ (without thermal stratification) and $\varepsilon=1$ (non-porous medium) are the same as those of Aziz and $\mathrm{Na}$ [13], Cebeci [16], and Minkowycz and Sparrow [17]. In the limiting case Eqs. (6)(8) govern the flow and heat transfer over a vertical plate if we omit the curvature term in the expressions $\left[(1+\xi \eta) f^{\prime \prime}\right]^{\prime}$ and $\left[(1+\xi \eta) \theta^{\prime}\right]^{\prime}$ of Eqs. (6) and (7). Also $(\xi / 2)$ should be replaced by $(\xi)^{1 / 4}$.

The local skin friction coefficient $C_{\mathrm{f} x}$ is given by

$$
C_{\mathrm{f} x}=2 \mu(\partial u / \partial r)_{r=r_{0}} / \rho U^{* 2}=2^{-1} G r_{x}^{-1 / 4} f^{\prime \prime}(\xi, 0) .
$$

Similarly, the local Nusselt number $N u_{x}$ can be expressed as;

$$
\begin{aligned}
N u_{x} & =-x(\partial T / \partial r)_{r=r_{0}} /\left(T_{\mathrm{w}}-T_{0}\right) \\
& =-G r_{x}^{1 / 4}\left[1-S(\xi / 2)^{4}\right]^{-1} \theta^{\prime}(\xi, 0)
\end{aligned}
$$

where $U^{*}=2 x^{1 / 2}\left[g \beta\left(T_{\mathrm{w}}-T_{0}\right)\right]^{1 / 2}$ is a hypothetical or equivalent velocity function and $\mu$ is the coefficient of viscosity.

\section{Perturbation method}

It is also possible to solve Eqs. (6) and (7) under the boundary conditions (8) by using a perturbation expansion procedure [13] in terms of the axial distance $\xi$. This approximate method is valid for small values of $\xi$, but its range of validity can be increased by applying the Shanks transformation [18]. In this method, one has to solve a system of ordinary differential equations instead of partial differential equations. At the same time it gives good results. We assume a regular perturbation expansion for $f$ and $\theta$ in powers of $\xi$ as given by;

$$
\begin{aligned}
& f(\xi, \eta)=\sum_{j=0}^{\infty} \xi^{j} f_{j}(\eta) \\
& \theta(\xi, \eta)=\sum_{j=0}^{\infty} \xi^{j} \theta_{j}(\eta) .
\end{aligned}
$$

Substituting (10) in Eqs. (6)-(8) and equating coefficients of like powers of $\xi$, we obtain.

$$
\text { For } j=0
$$

$\varepsilon^{-1} f_{0}^{\prime \prime \prime}+\varepsilon^{-2}\left(3 f_{0} f_{0}^{\prime \prime \prime}-f_{0}^{\prime 2}\right)+\theta_{0}=0$

$\theta_{0}^{\prime \prime}+3 \operatorname{Pr} f_{0} \theta_{0}^{\prime}=0$ 


$$
\begin{aligned}
& f_{0}(0)=f_{0}^{\prime}(0)=0, \quad \theta_{0}(0)=1 \\
& f_{0}^{\prime}(\infty)=\theta_{0}(\infty)=0 \\
& \quad \text { For } j=1, \text { we obtain } \\
& \varepsilon^{-1} f_{1}^{\prime \prime \prime}+\varepsilon^{-2}\left(3 f_{0} f_{1}^{\prime \prime}-2 f_{0}^{\prime} f_{1}^{\prime}-2 f_{0}^{\prime \prime} f_{1}\right) \\
& \quad+\varepsilon^{-1}\left(\eta f_{0}^{\prime \prime \prime}+f_{0}^{\prime \prime}\right)+\theta_{1} \\
& =\varepsilon^{-2}\left(f_{0}^{\prime} f_{1}^{\prime}-f_{0}^{\prime \prime} f_{1}\right) \\
& \theta_{1}^{\prime \prime}+3 \operatorname{Pr}\left(f_{0} \theta_{1}^{\prime}+\theta_{0}^{\prime} f_{1}\right)+\theta_{0}^{\prime}+\eta \theta_{0}^{\prime \prime} \\
& =\operatorname{Pr}\left(f_{0}^{\prime} \theta_{1}-\theta_{0}^{\prime} f_{1}\right) \\
& f_{1}(0)=f_{1}^{\prime}(0)=\theta_{1}(0)=f_{1}^{\prime}(\infty) \\
& \quad=\theta_{1}(\infty)=0
\end{aligned}
$$

For $j=2$, we have

$$
\begin{gathered}
\varepsilon^{-1} f_{2}^{\prime \prime \prime}+\varepsilon^{-2}\left[3\left(f_{0} f_{2}^{\prime \prime}+f_{1} f_{1}^{\prime \prime}+f_{2} f_{0}^{\prime \prime}\right)\right. \\
\left.-\left(2 f_{0}^{\prime} f_{2}^{\prime}+f_{1}^{\prime 2}\right)\right] \\
+\varepsilon^{-1}\left(\eta f_{1}^{\prime \prime \prime}+f_{1}^{\prime \prime}\right)+\theta_{2}-2^{-2} K^{-1} f_{0}^{\prime} \\
=\varepsilon^{-2}\left(2 f_{0}^{\prime} f_{2}^{\prime}+f_{1}^{\prime 2}-2 f_{0}^{\prime \prime} f_{2}-f_{0}^{\prime \prime} f_{1}\right) \\
\theta_{2}^{\prime \prime}+3 \operatorname{Pr}\left(f_{0} \theta_{2}^{\prime}+f_{1} \theta_{1}^{\prime}+f_{2} \theta_{0}^{\prime}\right)+\eta \theta_{1}^{\prime \prime}+\theta_{1}^{\prime} \\
=\operatorname{Pr}\left(2 f_{0}^{\prime} \theta_{2}+f_{1}^{\prime} \theta_{1}-2 \theta_{0}^{\prime} f_{2}-\theta_{1}^{\prime} f_{1}\right) \\
f_{2}(0)=f_{2}^{\prime}(0)=\theta_{2}(0)=f_{2}^{\prime}(\infty) \\
=\theta_{2}(\infty)=0
\end{gathered}
$$

For $j=3$, we get

$$
\begin{gathered}
\varepsilon^{-1} f_{3}^{\prime \prime \prime}+\varepsilon^{-2}\left[3\left(f_{0} f_{3}^{\prime \prime}+f_{1} f_{2}^{\prime \prime}+f_{2} f_{1}^{\prime \prime}+f_{3} f_{0}^{\prime \prime}\right)\right. \\
\left.\quad-2\left(f_{0}^{\prime} f_{3}^{\prime}+f_{1}^{\prime} f_{2}^{\prime}\right)\right] \\
+\varepsilon^{-1}\left(\eta f_{2}^{\prime \prime \prime}+f_{2}^{\prime \prime}\right)-2^{-2} K^{-1} f_{1}^{\prime}+\theta_{3} \\
=\varepsilon^{-2}\left[3\left(f_{0}^{\prime} f_{3}^{\prime}+f_{1}^{\prime} f_{2}^{\prime}\right)\right. \\
\left.\quad-\left(3 f_{0}^{\prime \prime} f_{3}+2 f_{1}^{\prime \prime} f_{2}+f_{2}^{\prime \prime} f_{1}\right)\right] \\
\theta_{3}^{\prime \prime}+3 \operatorname{Pr}\left(f_{0} \theta_{3}^{\prime}+f_{1} \theta_{2}^{\prime}+f_{2} \theta_{1}^{\prime}+f_{3} \theta_{0}^{\prime}\right)+\eta \theta_{2}^{\prime \prime}+\theta_{2}^{\prime} \\
=\operatorname{Pr}\left[\left(3 f_{0}^{\prime} \theta_{3}+2 f_{1}^{\prime} \theta_{2}+f_{2}^{\prime} \theta_{1}\right)\right. \\
\left.\quad-\left(3 \theta_{0}^{\prime} f_{3}+2 \theta_{1}^{\prime} f_{2}+\theta_{2}^{\prime} f_{1}\right)\right] \\
f_{3}(0)=f_{3}^{\prime}(0)=\theta_{3}(0)=f_{3}^{\prime}(\infty)=\theta_{3}(\infty)=0
\end{gathered}
$$

For $j=4$, the equations are given by

$$
\begin{aligned}
& \varepsilon^{-1} f_{4}^{\prime \prime \prime}+\varepsilon^{-2}[ 3\left(f_{0} f_{4}^{\prime \prime}+f_{1} f_{3}^{\prime \prime}+f_{2} f_{2}^{\prime \prime}\right. \\
&\left.+f_{3} f_{1}^{\prime \prime}+f_{4} f_{0}^{\prime \prime}\right) \\
&\left.-\left(2 f_{0}^{\prime} f_{4}^{\prime}+f_{1}^{\prime} f_{3}^{\prime}\right)-\left(f_{2}^{\prime}\right)^{2}\right] \\
&+\varepsilon^{-1}\left(\eta f_{3}^{\prime \prime \prime}+f_{3}^{\prime \prime}\right)+\theta_{4}-2^{-2} K^{-1} f_{2}^{\prime} \\
&-2^{-4} C\left(2 f_{0}^{\prime} f_{2}^{\prime}+f_{1}^{\prime 2}\right) \\
&+\varepsilon^{-2}\left[\left(4 f_{0}^{\prime} f_{4}^{\prime}+3 f_{1}^{\prime} f_{3}^{\prime}+2 f_{2}^{\prime 2}+f_{3}^{\prime} f_{1}^{\prime}\right)\right. \\
&\left.\quad-\left(4 f_{0}^{\prime \prime} f_{4}+3 f_{1}^{\prime \prime} f_{3}+2 f_{2}^{\prime \prime} f_{2}+f_{3}^{\prime \prime} f_{1}\right)\right]
\end{aligned}
$$

$$
\begin{aligned}
\theta_{4}^{\prime \prime}+ & 3 \operatorname{Pr}\left(f_{0} \theta_{4}^{\prime}+f_{1} \theta_{3}^{\prime}+f_{2} \theta_{2}^{\prime}+f_{3} \theta_{1}^{\prime}+f_{4} \theta_{0}^{\prime}\right) \\
+ & \eta \theta_{3}^{\prime \prime}+\theta_{3}^{\prime}-2^{-2} \operatorname{Pr} S f_{0}^{\prime} \\
=\operatorname{Pr} & {\left[\left(4 f_{0}^{\prime} \theta_{4}+3 f_{1}^{\prime} \theta_{3}+2 f_{2}^{\prime} \theta_{2}+f_{3}^{\prime} \theta_{1}\right)\right.} \\
& \left.\quad-\left(4 \theta_{0}^{\prime} f_{4}+3 \theta_{1}^{\prime} f_{3}+2 \theta_{2}^{\prime} f_{2}+\theta_{3}^{\prime} f_{1}\right)\right] \\
f_{4}(0)= & f_{4}^{\prime}(0)=0, \quad \theta_{4}(0)=-2^{-4} S \\
f_{4}^{\prime}(\infty)= & \theta_{4}(\infty)=0
\end{aligned}
$$

For $j \geqslant 5$, we obtain the following equations

$$
\begin{aligned}
& \varepsilon^{-1} f_{j}^{\prime \prime \prime}+\varepsilon^{-2}\left[\sum_{m=0}^{j}\left(3 f_{m} f_{j-m}^{\prime \prime}-f_{m}^{\prime} f_{j-m}^{\prime}\right)\right] \\
& +\varepsilon^{-1}\left(\eta f_{j-1}^{\prime \prime \prime}+f_{j-1}^{\prime \prime}\right)+\theta_{j}-2^{-2} K^{-1} f_{j-2}^{\prime} \\
& \quad-2^{-4} C \sum_{m=0}^{j-4} f_{m}^{\prime} f_{j-m-4}^{\prime} \\
& =\varepsilon^{-2} \sum_{m=0}^{j-1}(j-m)\left(f_{m}^{\prime} f_{j-m}^{\prime}-f_{m}^{\prime \prime} f_{j-m}\right) \\
& \theta_{j}^{\prime \prime}+3 \operatorname{Pr} \sum_{m=0}^{j} f_{m} \theta_{j-m}^{\prime}+\eta \theta_{j-1}^{\prime \prime}+\theta_{j-1}^{\prime}-4 \operatorname{Pr} S f_{j-4}^{\prime} \\
& =\operatorname{Pr} \sum_{m=0}^{j-1}(j-m)\left(f_{m}^{\prime} \theta_{j-m}-\theta_{m} f_{j-m}\right) \\
& f_{j}(0)=f_{j}^{\prime}(0)=\theta_{j}(0)=f_{j}^{\prime}(\infty)=\theta_{j}(\infty)=0 .
\end{aligned}
$$

The above system of equations is linear except (11a) and (11b) which are nonlinear. Hence Eqs. (11a) and (11b) are solved using the shooting method [19] and the subsequent equations are solved by employing the method of superposition [19]. The local skin friction parameter $\left(f^{\prime \prime}(\xi, 0)\right)$ and the local heat transfer parameter $\left(\theta^{\prime}(\xi, 0)\right)$ can be expressed as;

$$
\begin{aligned}
& f^{\prime \prime}(\xi, 0)=\sum_{j=0}^{\infty} \xi^{j} f_{j}^{\prime \prime}(0) \\
& \theta^{\prime}(\xi, 0)=\sum_{j=0}^{\infty} \xi^{j} \theta_{j}^{\prime}(0) .
\end{aligned}
$$

Eq. (17) is valid for small values of $\xi(\xi \ll 1)$, but its range of validity can be increased by applying the Shanks transformation [18]

$$
e\left(L_{j}\right)=\left(L_{j+1} L_{j-1}-L_{j}^{2}\right) /\left(L_{j+1}+L_{j-1}-2 L_{j}\right)
$$

where $e$ is an operator and $L_{j}$ is the partial sum. Here we have taken $j=5$ and applied the transformation (18) to Eqs. (17) twice and we find that the results obtained are in very good agreement with the numerical results. The difference is less than 1 per cent in the range $0 \leqslant \xi \leqslant$ 1 , and about $2 \%$ in the range $1 \leqslant \xi \leqslant 1.5$. The surface shear stress and the heat transfer $\left[\left(f_{j}^{\prime \prime}(0), \theta_{j}^{\prime}(0)\right]_{j=0,1,2,3,4,5}\right.$ obtained from Eqs. (11)-(16) are given in Tables 1 and 2. The application of Shanks transformations (18) to the series 
Table 1

Surface shear stress values for $\operatorname{Pr}=0.7, \varepsilon=0.9$

\begin{tabular}{|c|c|c|c|c|c|c|c|c|}
\hline$S$ & $C$ & $K$ & $f_{0}^{\prime}(0)$ & $f_{1}^{\prime}(0)$ & $f_{2}^{\prime}(0)$ & $f_{3}^{\prime}(0)$ & $f_{4}^{\prime}(0)$ & $f_{5}^{\prime}(0)$ \\
\hline 0 & 0 & 0.316 & $6.514 \mathrm{E}-1$ & $2.252 \mathrm{E}-1$ & $-2.026 \mathrm{E}-1$ & $3.045 \mathrm{E}-1$ & $-5.076 \mathrm{E}-1$ & 7.478 \\
\hline 0.25 & 0 & 0.316 & $6.514 \mathrm{E}-1$ & $2.252 \mathrm{E}-1$ & $-2.026 \mathrm{E}-1$ & $3.045 \mathrm{E}-1$ & $-5.156 \mathrm{E}-1$ & 7.429 \\
\hline 0.5 & 0 & 0.316 & $6.514 \mathrm{E}-1$ & $2.252 \mathrm{E}-1$ & $-2.026 \mathrm{E}-1$ & $3.045 \mathrm{E}-1$ & $-5.235 \mathrm{E}-1$ & 7.381 \\
\hline 0.75 & 0 & 0.316 & $6.514 \mathrm{E}-1$ & $2.252 \mathrm{E}-1$ & $-2.026 \mathrm{E}-1$ & $3.045 \mathrm{E}-1$ & $-5.314 \mathrm{E}-1$ & 7.332 \\
\hline 1.0 & 0 & 0.316 & $6.514 \mathrm{E}-1$ & $2.252 \mathrm{E}-1$ & $-2.026 \mathrm{E}-1$ & $3.045 \mathrm{E}-1$ & $-5.393 \mathrm{E}-1$ & 7.284 \\
\hline 0 & 0 & 1.0 & $6.514 \mathrm{E}-1$ & $2.252 \mathrm{E}-1$ & $-1.425 \mathrm{E}-1$ & $3.543 \mathrm{E}-1$ & $-5.713 \mathrm{E}-1$ & 7.767 \\
\hline 0.25 & 0 & 1.0 & $6.514 \mathrm{E}-1$ & $2.252 \mathrm{E}-1$ & $-1.425 \mathrm{E}-1$ & $3.543 \mathrm{E}-1$ & $-5.792 \mathrm{E}-1$ & 7.718 \\
\hline 0.5 & 0 & 1.0 & $6.514 \mathrm{E}-1$ & $2.252 \mathrm{E}-1$ & $-1.425 \mathrm{E}-1$ & $3.543 \mathrm{E}-1$ & $-5.872 \mathrm{E}-1$ & 7.670 \\
\hline 0.75 & 0 & 1.0 & $6.514 \mathrm{E}-1$ & $2.252 \mathrm{E}-1$ & $-1.425 \mathrm{E}-1$ & $3.543 \mathrm{E}-1$ & $-5.951 \mathrm{E}-1$ & 7.621 \\
\hline 1.0 & 0 & 1.0 & $6.514 \mathrm{E}-1$ & $2.252 \mathrm{E}-1$ & $-1.425 \mathrm{E}-1$ & $3.543 \mathrm{E}-1$ & $-6.030 \mathrm{E}-1$ & 7.573 \\
\hline 0 & 200 & 0.316 & $6.514 \mathrm{E}-1$ & $2.252 \mathrm{E}-1$ & $-2.026 \mathrm{E}-1$ & $3.045 \mathrm{E}-1$ & $-1.937 \mathrm{E}-1$ & 7.311 \\
\hline 0.25 & 200 & 0.316 & $6.514 \mathrm{E}-1$ & $2.252 \mathrm{E}-1$ & $-2.026 \mathrm{E}-1$ & $3.045 \mathrm{E}-1$ & $-2.016 \mathrm{E}-1$ & 7.262 \\
\hline 0.5 & 200 & 0.316 & $6.514 \mathrm{E}-1$ & $2.252 \mathrm{E}-1$ & $-2.026 \mathrm{E}-1$ & $3.045 \mathrm{E}-1$ & $-2.095 \mathrm{E}-1$ & 7.214 \\
\hline 0.75 & 200 & 0.316 & $6.514 \mathrm{E}-1$ & $2.252 \mathrm{E}-1$ & $-2.026 \mathrm{E}-1$ & $3.045 \mathrm{E}-1$ & $-2.174 \mathrm{E}-1$ & 7.165 \\
\hline 0 & 200 & 1.0 & $6.514 \mathrm{E}-1$ & $2.252 \mathrm{E}-1$ & $-1.425 \mathrm{E}-1$ & $3.543 \mathrm{E}-1$ & $-3.138 \mathrm{E}-1$ & 7.590 \\
\hline 0.25 & 200 & 1.0 & $6.514 \mathrm{E}-1$ & $2.252 \mathrm{E}-1$ & $-1,425 \mathrm{E}-1$ & $3.543 \mathrm{E}-1$ & $-3.217 \mathrm{E}-1$ & 7.542 \\
\hline 0.50 & 200 & 1.0 & $6.514 \mathrm{E}-1$ & $2.252 \mathrm{E}-1$ & $-1.425 \mathrm{E}-1$ & $3.543 \mathrm{E}-1$ & $-3.296 \mathrm{E}-1$ & 7.493 \\
\hline 0.75 & 200 & 1.0 & $6.514 \mathrm{E}-1$ & $2.252 \mathrm{E}-1$ & $-1.425 \mathrm{E}-1$ & $3.543 \mathrm{E}-1$ & $-3.375 \mathrm{E}-1$ & 7.445 \\
\hline 1.0 & 200 & 1.0 & $6.514 \mathrm{E}-1$ & $2.252 \mathrm{E}-1$ & $-1.425 \mathrm{E}-1$ & $3.543 \mathrm{E}-1$ & $-3.454 \mathrm{E}-1$ & 7.396 \\
\hline
\end{tabular}

Table 2

Surface heat transfer values for $\operatorname{Pr}=0.7, \varepsilon=0.9$

\begin{tabular}{|c|c|c|c|c|c|c|c|c|}
\hline$S$ & $C$ & $K$ & $\theta_{0}^{\prime}(0)$ & $\theta_{1}^{\prime}(0)$ & $\theta_{2}^{\prime}(0)$ & $\theta_{3}^{\prime}(0)$ & $\theta_{4}^{\prime}(0)$ & $\theta_{5}^{\prime}(0)$ \\
\hline 0 & 0 & 0.316 & $4.950 \mathrm{E}-1$ & $1.170 \mathrm{E}-1$ & $-1.479 \mathrm{E}-1$ & $4.055 \mathrm{E}-1$ & -3.188 & 9.352 \\
\hline 0.25 & 0 & 0.316 & $4.950 \mathrm{E}-1$ & $1.170 \mathrm{E}-1$ & $-1.479 \mathrm{E}-1$ & $4.055 \mathrm{E}-1$ & -3.194 & 9.419 \\
\hline 0.5 & 0 & 0.316 & $4.950 \mathrm{E}-1$ & $1.170 \mathrm{E}-1$ & $-1.479 \mathrm{E}-1$ & $4.055 \mathrm{E}-1$ & -3.200 & 9.488 \\
\hline 0.75 & 0 & 0.316 & $4.950 \mathrm{E}-1$ & $1.170 \mathrm{E}-1$ & $-1.479 \mathrm{E}-1$ & $4.055 \mathrm{E}-1$ & -3.207 & 9.555 \\
\hline 1.0 & 0 & 0.316 & $4.950 \mathrm{E}-1$ & $1.170 \mathrm{E}-1$ & $-1.479 \mathrm{E}-1$ & $4.055 \mathrm{E}-1$ & -3.213 & 9.622 \\
\hline 0 & 0 & 1.0 & $4.950 \mathrm{E}-1$ & $1.170 \mathrm{E}-1$ & $-1.479 \mathrm{E}-1$ & $4.055 \mathrm{E}-1$ & -3.276 & 9.631 \\
\hline 0.25 & 0 & 1.0 & $4.950 \mathrm{E}-1$ & $1.170 \mathrm{E}-1$ & $-1.479 \mathrm{E}-1$ & $4.055 \mathrm{E}-1$ & -3.282 & 9.699 \\
\hline 0.5 & 0 & 1.0 & $4.950 \mathrm{E}-1$ & $1.170 \mathrm{E}-1$ & $-1.479 \mathrm{E}-1$ & $4.055 \mathrm{E}-1$ & -3.288 & 9.766 \\
\hline 0.75 & 0 & 1.0 & $4.950 \mathrm{E}-1$ & $1.170 \mathrm{E}-1$ & $-1.479 \mathrm{E}-1$ & $4.055 \mathrm{E}-1$ & -3.294 & 9.834 \\
\hline 1.0 & 0 & 1.0 & $4.950 \mathrm{E}-1$ & $1.170 \mathrm{E}-1$ & $-1.479 \mathrm{E}-1$ & $4.055 \mathrm{E}-1$ & -3.301 & 9.901 \\
\hline 0 & 200 & 0.316 & $4.950 \mathrm{E}-1$ & $1.170 \mathrm{E}-1$ & $-1.479 \mathrm{E}-1$ & $4.055 \mathrm{E}-1$ & -3.061 & 9.232 \\
\hline 0.25 & 200 & 0.316 & $4.950 \mathrm{E}-1$ & $1.170 \mathrm{E}-1$ & $-1.479 \mathrm{E}-1$ & $4.055 \mathrm{E}-1$ & -3.067 & 9.299 \\
\hline 0.5 & 200 & 0.316 & $4.950 \mathrm{E}-1$ & $1.170 \mathrm{E}-1$ & $-1.479 \mathrm{E}-1$ & $4.055 \mathrm{E}-1$ & -3.073 & 9.367 \\
\hline 0.75 & 200 & 0.316 & $4.950 \mathrm{E}-1$ & $1.170 \mathrm{E}-1$ & $-1.479 \mathrm{E}-1$ & $4.055 \mathrm{E}-1$ & -3.079 & 9.434 \\
\hline 0 & 200 & 1.0 & $4.950 \mathrm{E}-1$ & $1.170 \mathrm{E}-1$ & $-1.479 \mathrm{E}-1$ & $4.055 \mathrm{E}-1$ & -3.177 & 9.520 \\
\hline 0.25 & 200 & 1.0 & $4.950 \mathrm{E}-1$ & $1.170 \mathrm{E}-1$ & $-1.479 \mathrm{E}-1$ & $4.055 \mathrm{E}-1$ & -3.183 & 9.588 \\
\hline 0.50 & 200 & 1.0 & $4.950 \mathrm{E}-1$ & $1.170 \mathrm{E}-1$ & $-1.479 \mathrm{E}-1$ & $4.055 \mathrm{E}-1$ & -3.190 & 9.655 \\
\hline 0.75 & 200 & 1.0 & $4.950 \mathrm{E}-1$ & $1.170 \mathrm{E}-1$ & $-1.479 \mathrm{E}-1$ & $4.055 \mathrm{E}-1$ & -3.196 & 9.723 \\
\hline 1.0 & 200 & 1.0 & $4.950 \mathrm{E}-1$ & $1.170 \mathrm{E}-1$ & $-1.479 \mathrm{E}-1$ & $4.055 \mathrm{E}-1$ & -3.202 & 9.790 \\
\hline
\end{tabular}

given by Eqs. (17) for certain values of the parameters is illustrated in Table 3. Some of the results thus obtained are compared with those obtained by using the finite difference method and this comparison is shown in Figs. 6 and 7.

\section{Numerical method}

The coupled nonlinear partial differential Eqs. (6) and (7) under the boundary conditions (8) are solved numerically using an implicit, iterative, tri-diagonal finite-difference scheme similar to that discussed by Blottner [12]. The first-
Table 3

Application of Shanks transformation (18) to the series given by $\theta^{\prime}(\xi, 0)=$ $\sum \xi^{j} \theta^{\prime}(0)$, in (17) for $\xi=1, S=0.25, \operatorname{Pr}=0.7, \varepsilon=0.9, K=1$, and $C=200$

\begin{tabular}{lccc}
\hline$J$ & $L_{j}$ & $E$ & $e^{2}$ \\
\hline 0 & -0.4950 & & \\
1 & -0.6120 & -0.5536 & \\
2 & -0.4956 & -0.5874 & -0.5690 \\
3 & -0.9293 & -0.5476 & \\
4 & -2.2537 & & \\
\hline
\end{tabular}


order derivatives with respect to $\xi$ are replaced by two-point backward difference formulae of the form

$\partial R / \partial \xi=\left(R_{m, n}-R_{m-1, n}\right) / \Delta \xi$

where $R$ stands for the dependent variable $f$ or $\theta$ and $m$ and $n$ are the node locations along the $\xi$ and $\eta$ directions, respectively. First the third-order Eq. (6) is converted into a second-order by substituting $f^{\prime}=F$. Then the second-order partial differential equations for $F$ and $\theta$ are discretized using the three-point central difference formulae and the firstorder equations are discretized by employing the trapezoidal rule. At each line of constant $\xi$, we get a system of algebraic equations. We evaluate the nonlinear terms in the equations at the previous iteration and solve the system of algebraic equations interatively by using the Thomas algorithm (see Blottner [12]). The same procedure is repeated for the next $\xi$ value and the equations are solved line by line until the desired $\xi$ value is reached. A convergence criterion based on the relative difference between the current and the previous iterations is employed. When this difference reaches $10^{-5}$, the solution is assumed to have converged and the iterative process is terminated.

We have varied the grid size $\Delta \xi$ and $\Delta \eta$. For grid size less than $60 \times 100$, the numerical values differ only in the fourth decimal place. This corresponds to an error of less than $0.5 \%$. We have chosen the grid size $120 \times 200$ for the results presented.

\section{Results and discussion}

Eqs. (6) and (7) under the boundary conditions (8) have been solved numerically using the method described in the previous section. The results have been presented for various values of the ambient thermal stratification parameter $S(0 \leqslant S \leqslant 0.96)$, inertia parameter $C(0,100,200)$, permeability parameter $K(0.316,1)$, Prandtl number $\operatorname{Pr}(0.7,5.4$, 7.0) and the curvature parameter $\xi(0 \leqslant \xi \leqslant 3)$.

In order to assess the accuracy of the method, we have compared the ratio of local Nusselt numbers, $N u_{x} /$
$\left(N u_{x}\right)_{\xi=0}$, for $S=C=K^{-1}=0, \varepsilon=1$ (obtained by using the finite-difference method) with of the finite-difference results of Cebeci [16], the local non-similarity results of Minkowycz and Sparrow [17] and the perturbation expansion method (with Shanks transformation) results of Aziz and $\mathrm{Na}[13]$. The results differ by less than $0.5 \%$ from the finite-difference results of Ref. [16] and about $1 \%$ from the local non-similarity results of Ref. [17] and the results of the perturbation method of Ref. [13] when $\xi=4$. This difference decreases with decreasing $\xi$. The comparison is given in Table 4.

We have also compared the heat transfer parameter $-\theta^{\prime}(\xi, 0)$ for the vertical plate in a stratified fluid in a uniform medium $(\varepsilon=1)$ for $K^{-1}=C=0$, with that of Eichhorn [6], Chen and Eichhorn [7], and Venkatachala and Nath [8], who used the series method, local non-similarity method and the finite difference method respectively. Since the results of Venkatachala and Nath [8] are found to be identical (at least up to the 3rd decimal place), these are not shown in the Fig. 2. For large $\operatorname{Pr}(\operatorname{Pr}=6)$ the local nonsimilarity results of Chen and Eichhorn [7] are in very good agreement with the present results obtained by using the finite-difference method. However, for small $\operatorname{Pr}(\operatorname{Pr}=0.7)$ and for $\xi \geqslant 0.6$, the local non-similarity method slightly over-estimates the heat transfer. On the other hand, the series solution results of Eichhorn [6] are in in good agreement for $\xi \leqslant 0.6$, but beyond this value it may differ significantly. The series solution for $\xi \geqslant 0.6$ under-estimates the heat transfer results. Further, we have compared the value of the average Nusselt number for the plate

$G r_{x}^{-1 / 4} \overline{N u}=-\int_{0}^{1}(1-S \xi)^{-1} \theta^{\prime}(\xi, 0) \mathrm{d} \xi$

for $0 \leqslant S<1, \operatorname{Pr}=5.4, K=0.316,1, C=0,200, \varepsilon=$ 0.9 with that of Chen and Lin [4] and found them to be in good agreement. This comparison is shown in Fig. 3.

The effect of the ambient thermal stratification parameter $S$ on the local skin friction coefficient $\left(2 G r_{x}^{1 / 4} C_{\mathrm{f} x}\right)$ and the

Table 4

Comparison of the ratio of local Nusselt numbers, $N u_{x} /\left(N u_{x}\right)_{\xi=0}$ for $S=C=K^{-1}=0, \varepsilon=1$

$$
N u_{x} /\left(N u_{x}\right)_{\xi=0}
$$

\begin{tabular}{lllcc}
\hline $\operatorname{Pr}$ & $\xi$ & Present & Aziz and Na [13] & Cebeci [16] \\
\hline 0.72 & 0 & 1.000 & 1.000 & 1.000 \\
0.72 & 0.503 & 1.210 & 1.219 & 1.210 \\
0.72 & 1.064 & 1.424 & 1.445 & 1.422 \\
0.72 & 2.093 & 1.781 & 1.821 & 1.778 \\
0.72 & 3.364 & 2.183 & 2.232 & 2.177 \\
0.72 & 4.000 & 2.378 & 2.419 & 1.366 \\
10 & 0 & 1.000 & 1.000 & 1.000 \\
10 & 0.503 & 1.096 & 1.096 & 1.096 \\
10 & 1.064 & 1.196 & 1.196 & 1.196 \\
10 & 2.093 & 1.371 & 1.367 & 1.373 \\
10 & 3.364 & 1.569 & 1.650 & - \\
10 & 4.000 & 1.665 & - & 1.575 \\
\hline
\end{tabular}




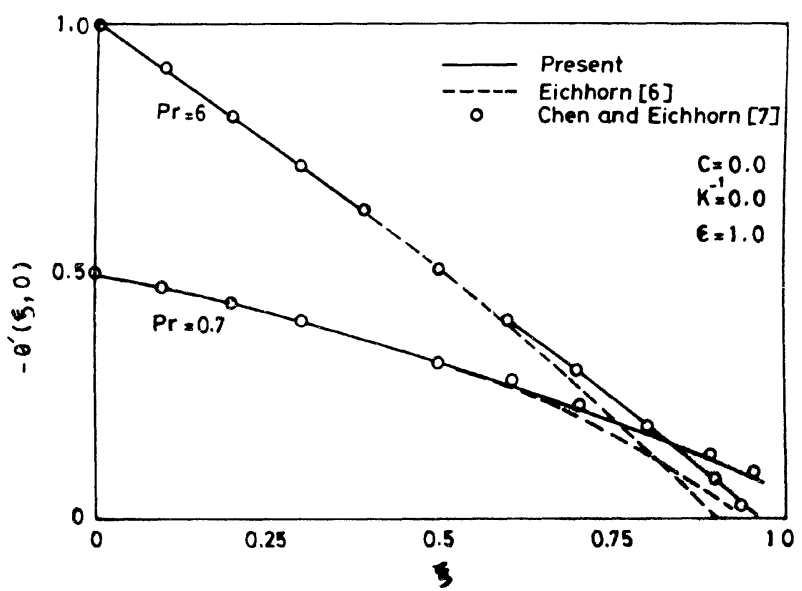

Fig. 2. Comparison of heat transfer parameter $-\theta^{\prime}(\xi, 0)$ for the plate when $\varepsilon=S=1, K^{-1}=C=0, \operatorname{Pr}=0.7,6.0$.

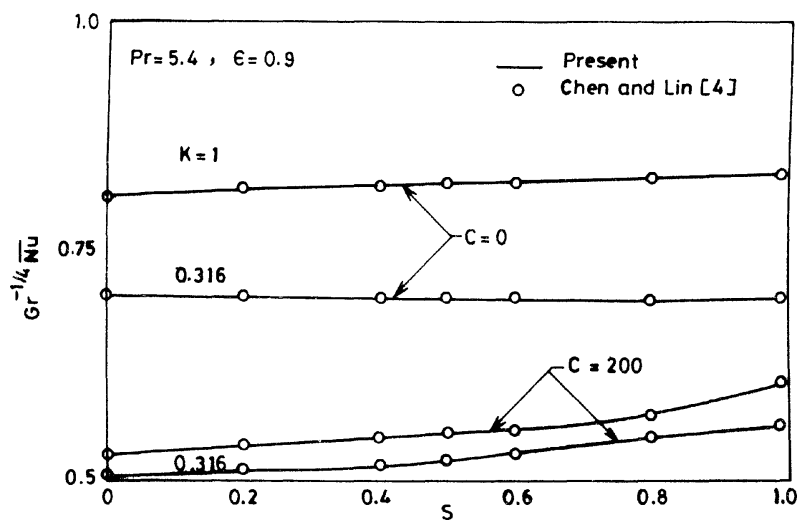

Fig. 3. Comparison of the average Nusselt number $G r_{x}^{-1 / 4} \overline{N u}$ for the vertical plate when $\varepsilon=0.9, \operatorname{Pr}=5.4, K=0.316,1, C=0,200$.

local Nusselt number $\mathrm{Gr}_{x}^{-1 / 4} \mathrm{Nu}$ x for $\mathrm{Pr}=0.7,5.4,7.0$, $C=200, K=1, \varepsilon=0.9, \xi=2$ is shown in Figs. 4 and 5 . An increase in the thermal stratification parameter, generally, decreases the thermal buoyancy. Hence the velocity and temperature and their gradients reduce with increasing $S$. This results in lower skin friction and heat transfer. It is also observed that the skin friction coefficient for $\mathrm{Pr}=$ 0.7 vanishes for $S=0.96$. Since boundary layer equations are not valid beyond zero skin friction, we have confined our study in the range $0 \leqslant S \leqslant 0.96$. For $\operatorname{Pr}=0.7$, the local Nusselt number becomes negative for $S>0.714$. This implies that the temperature of the fluid near the wall exceeds the wall temperature. For $S<0.7$ the skin friction decreases as the Prandtl number increases, but for $S>0.7$ it increases. However, an opposite trend is observed for the Nusselt number. For this case $S=0.6$ instead of 0.7 .

The effect of the curvature parameter $\xi$ on the local skin friction coefficient and the local Nusselt number $\left(2 G r_{x}^{1 / 4} C_{\mathrm{f} x}, G r_{x}^{-1 / 4} N u_{x}\right)$ for $\operatorname{Pr}=0.7,5.4,7.0, C=200$, $K=1, S=0.2, \varepsilon=0.9$ is presented in Figs. 6 and 7 . The corresponding results obtained by using the perturbation technique with the Shanks transformation are also shown in the figures. It is observed that for stratified fluid both the

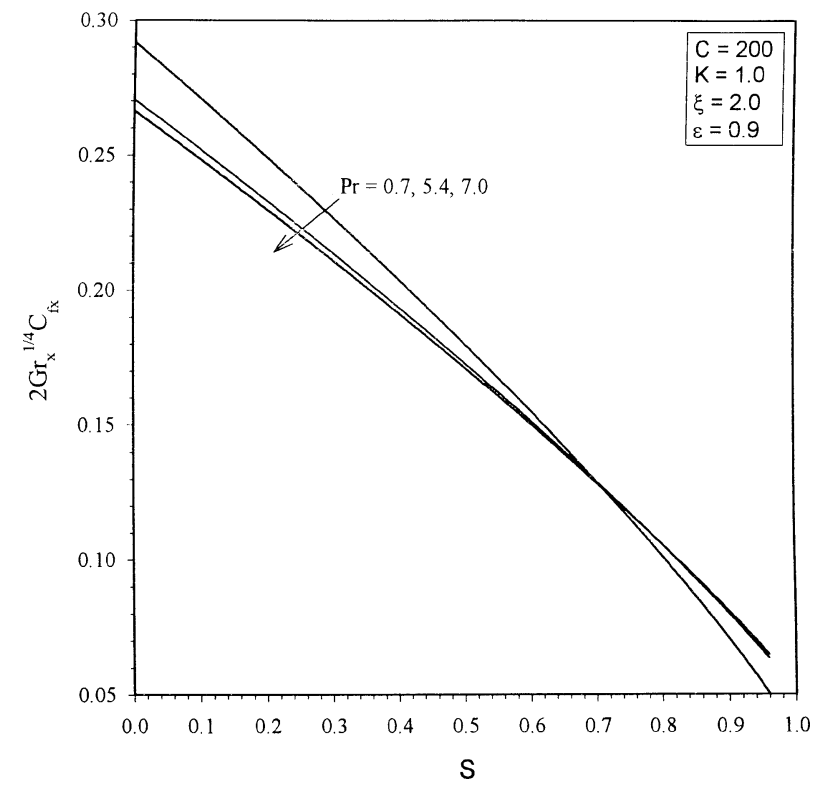

Fig. 4. Effect of the ambient thermal stratification parameter $S$ on the local skin friction coefficient, $2 G r_{x}^{1 / 4} C_{\mathrm{f} x}$.

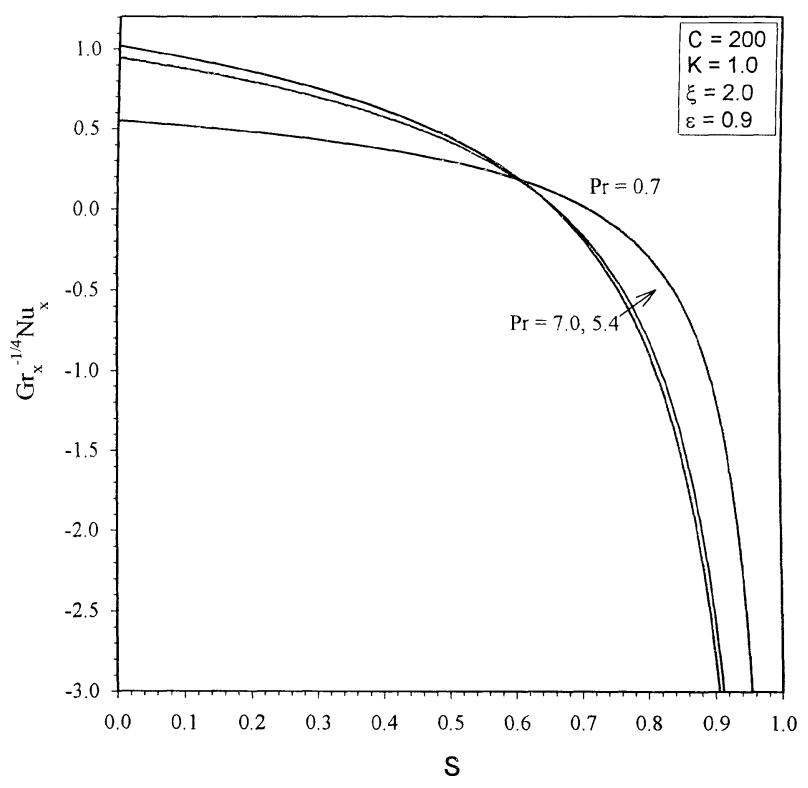

Fig. 5. Effect of the ambient thermal stratification parameter $S$ on the local Nusselt number, $G r_{x}^{-1 / 4} N u_{x}$.

skin friction and the Nusselt number decrease with increasing curvature due to the thickening of momentum and thermal boundary layers. The effect of $\xi$ is more pronounced for large $\xi$. The results of the perturbation analysis for $0 \leqslant \xi \leqslant 2$ are found to be in very good agreement with those obtained by using the finite difference method. These differ by about $0.5 \%$ for $\xi=2$ and this difference decreases with decreasing $\xi$.

Fig. 8 shows the effect of the ambient thermal stratification parameter $S$ on the velocity and temperature profiles $\left(f^{\prime}(\xi, \eta), \theta(\xi, \eta)\right)$ for $\xi=2, \varepsilon=0.9, C=200, K=$ $1, \operatorname{Pr}=5.4$. Both the velocity and temperature profiles de- 


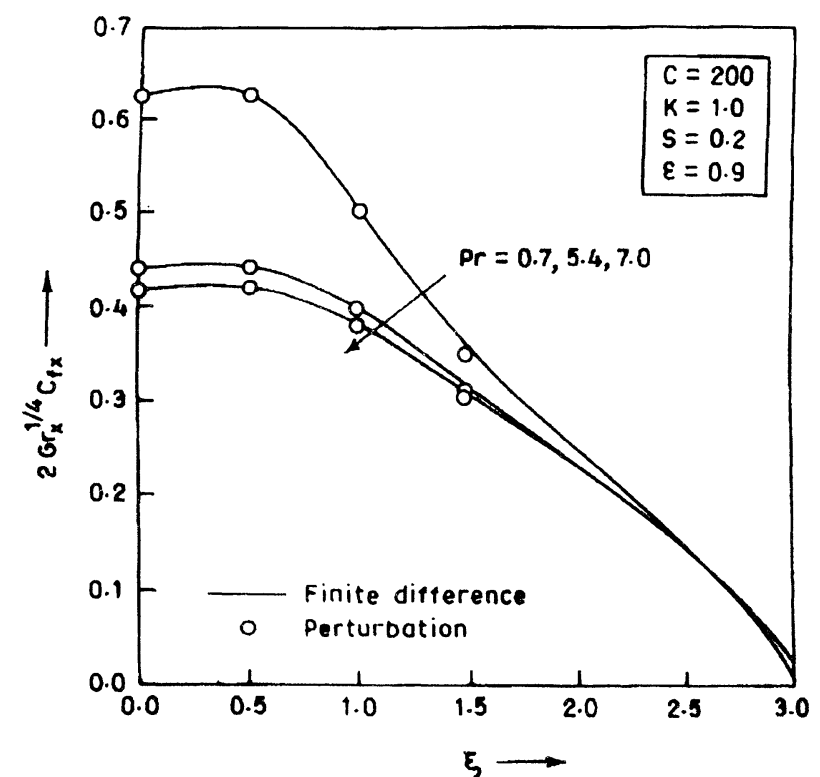

Fig. 6. Effect of the curvature parameter $\xi$ on the local skin friction coefficient $2 G r_{x}^{1 / 4} C_{\mathrm{f} x}$.

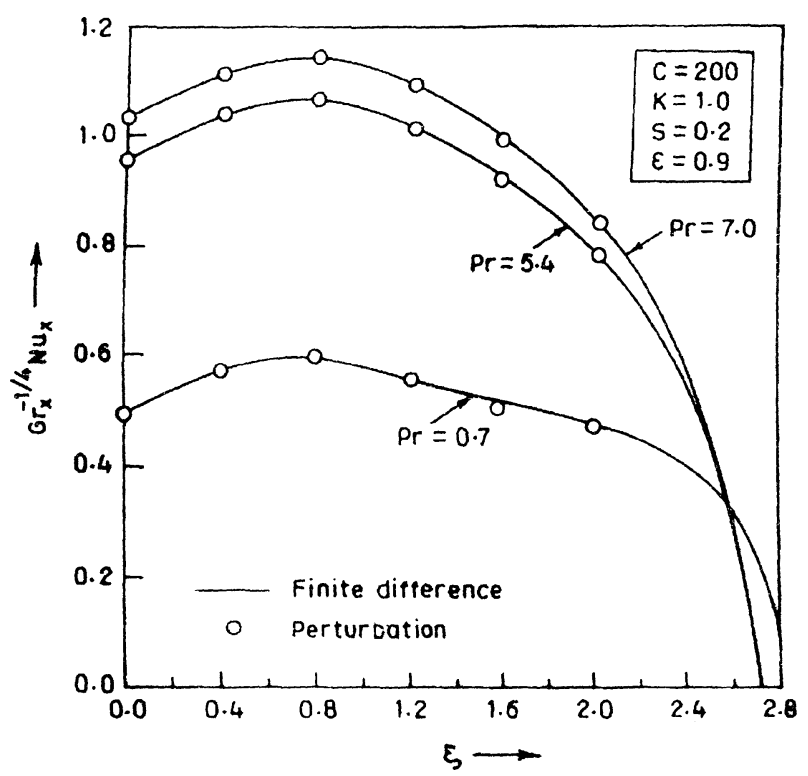

Fig. 7. Effect of the curvature parameter $\xi$ on the local Nusselt number, $G r_{x}^{-1 / 4} N u_{x}$.

crease with increasing $S$ since an increase in $S$ implies a reduction in the thermal buoyancy. The effect of $S$ is more pronounced on the temperature profiles than on the velocity profiles. The reason for this trend is that the thermal stratification parameter $S$ explicitly occurs in the energy equation as well as in the boundary conditions, whereas its effect on the velocity profiles is indirect (see Eqs. (6)-(8)).

In Fig. 9 the effect of the curvature parameter $\xi$ on the velocity and temperature profiles $\left(f^{\prime}(\xi, \eta), \theta(\xi, \eta)\right)$ for $S=$ $0.5, C=200, K=1, \operatorname{Pr}=5.4, \varepsilon=0.9$ is displayed. The peak velocity decreases with increasing curvature parameter due to the increase in the momentum boundary layer

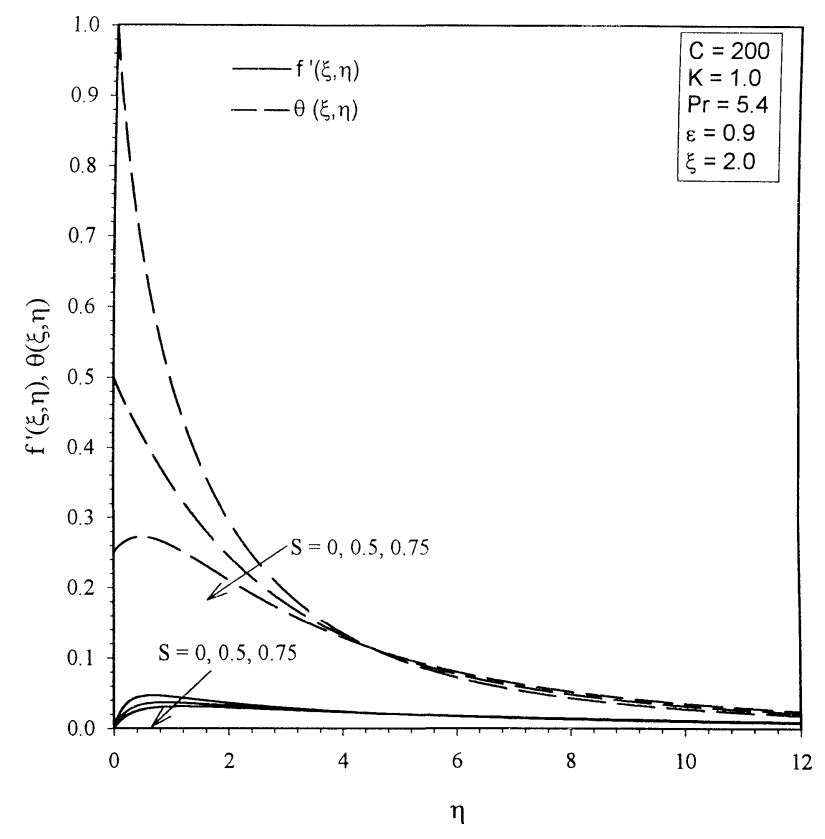

Fig. 8. Effects of the ambient thermal stratification parameter $S$ on the velocity and temperature profiles, $f^{\prime}(\xi, \eta), \theta(\xi, \eta)$.

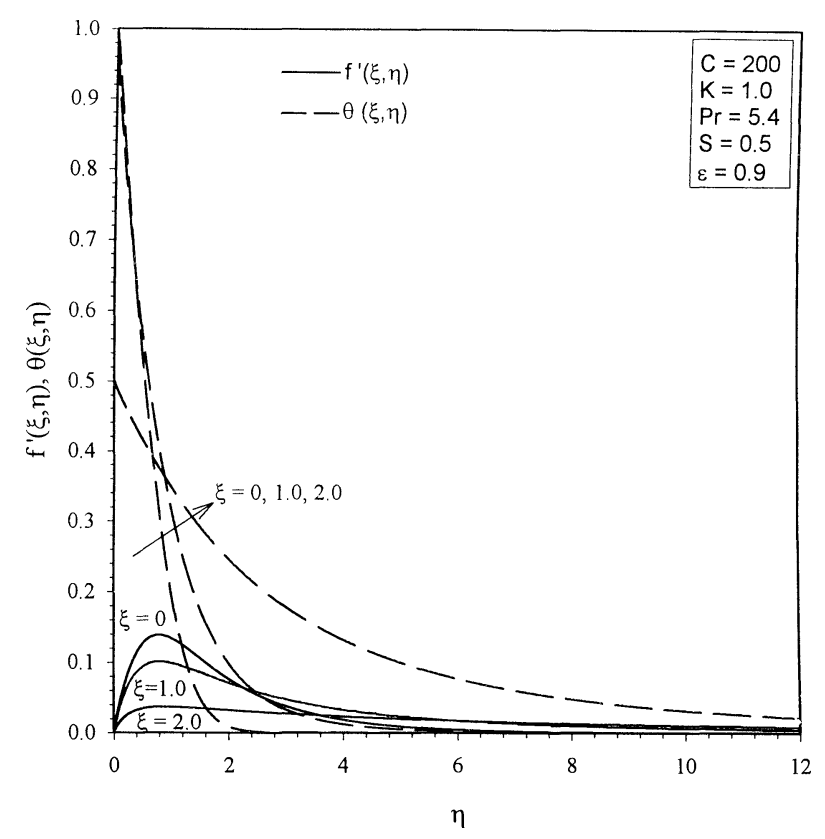

Fig. 9. Effects of the curvature parameter $\xi$ on the velocity and temperature profiles, $f^{\prime}(\xi, \eta), \theta(\xi, \eta)$.

thickness. The temperature profiles increase with $\xi$ (except near the wall) since the thermal boundary layer increases.

Fig. 10 presents the effect of the inertia parameter $C$ on the velocity and temperature profiles $\left(f^{\prime}(\xi, \eta), \theta(\xi, \eta)\right)$ for $S=0.5, K=1, P r=5.4, \varepsilon=0.9, \xi=2$. The increase in the inertia parameter implies more resistance to the flow which results in an increase in the momentum boundary layer and hence in the thermal boundary layer. Consequently, the peak velocity decreases and the temperature increases as $C$ increases. 


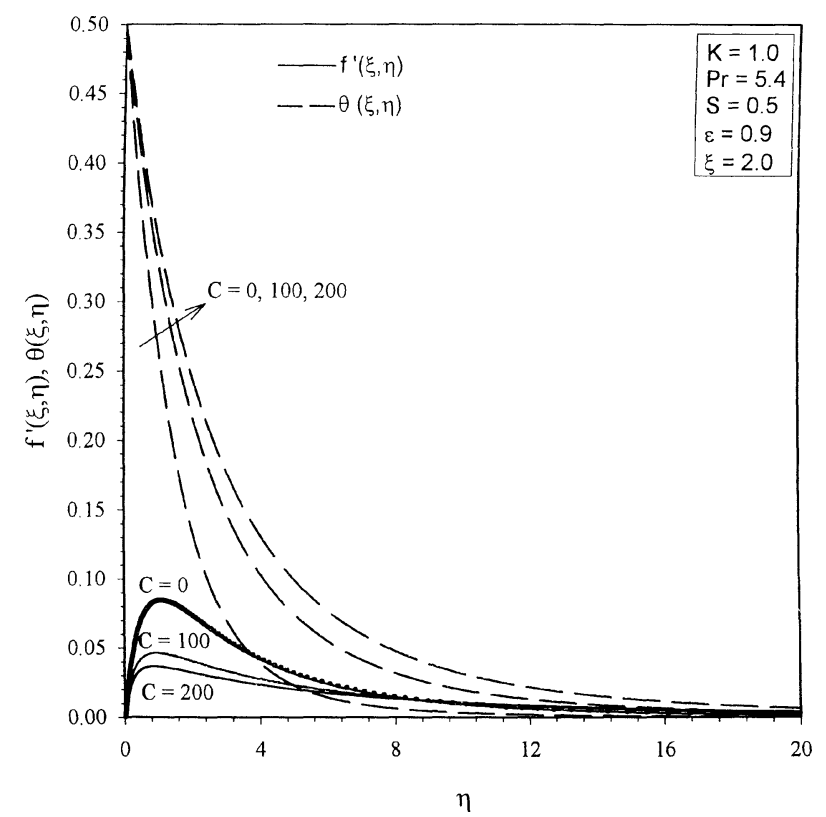

Fig. 10. Effects of the inertia parameter $C$ on the velocity and temperature profiles, $f^{\prime}(\xi, \eta), \theta(\xi, \eta)$.

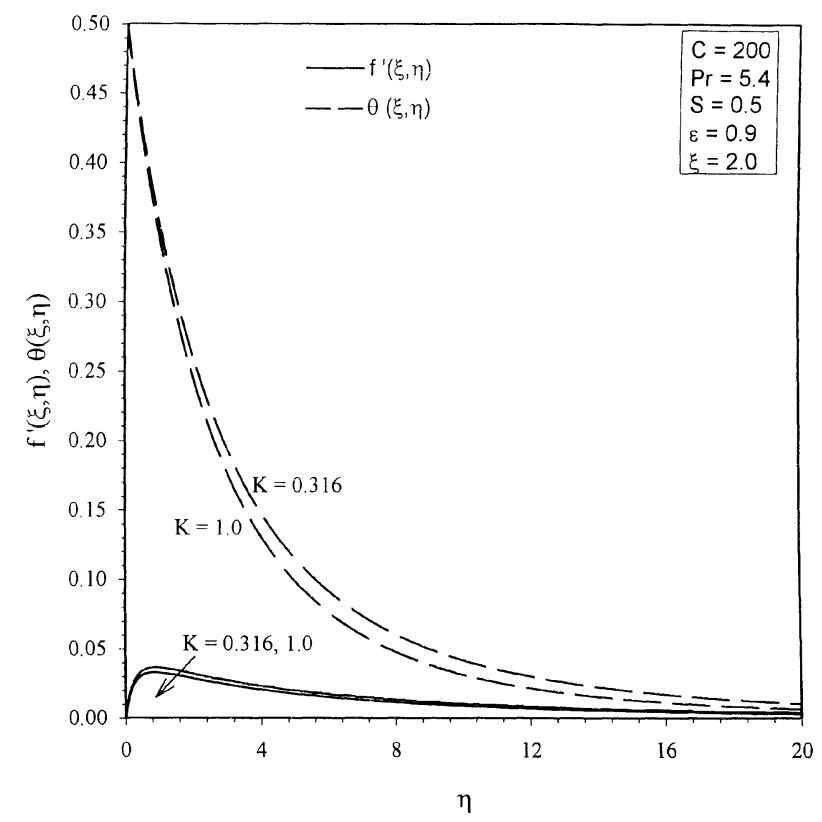

Fig. 11. Effects of the permeability parameter $K$ on the velocity and temperature profiles, $f^{\prime}(\xi, \eta), \theta(\xi, \eta)$.

The effect of the permeability parameter $K$ on the velocity and temperature profiles $\left(f^{\prime}(\xi, \eta), \theta(\xi, \eta)\right)$ for $C=$ 200, $S=0.5, \operatorname{Pr}=5.4, \varepsilon=0.9$, and $\xi=2$ is shown in Fig. 11. It is observed that the temperature and the peak velocity reduce as $K$ increases which implies that the resistance of the medium decreases.

Fig. 12 presents the effect of the Prandtl number $\operatorname{Pr}$ on the velocity and temperature profiles $\left(f^{\prime}(\xi, \eta), \theta(\xi, \eta)\right)$ for $C=200, K=1, \quad S=0.5, \varepsilon=0.9, \quad \xi=2$. Since the higher Prandtl number $\mathrm{Pr}$ implies lower effective thermal

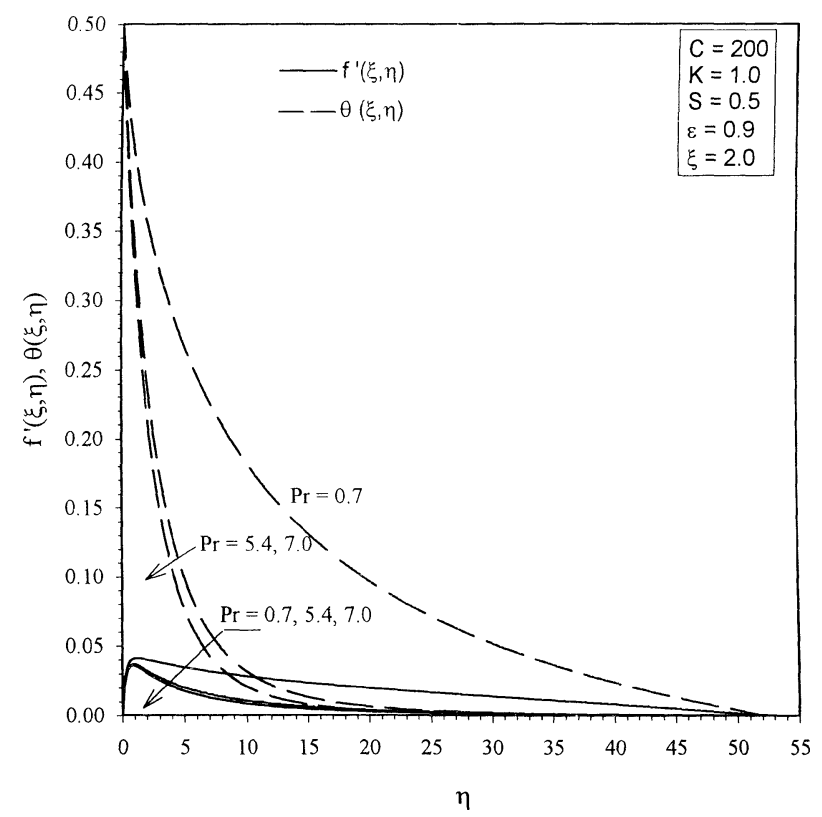

Fig. 12. Effects of the Prandtl number $\operatorname{Pr}$ on the velocity and temperature profiles, $f^{\prime}(\xi, \eta), \theta(\xi, \eta)$.

Table 5

Local skin friction coefficient $\left(2 G r_{x}^{1 / 4} C_{\mathrm{f} x}\right)$ and local Nusselt number $\left(G r_{x}^{-1 / 4} N u_{x}\right)$ for $K=1.0, S=0.5, \varepsilon=0.9$ and $\xi=2.0$

\begin{tabular}{rlcc}
\hline$C$ & $P r$ & $2 G r_{x}^{1 / 4} C_{\mathrm{f} x}$ & $G r_{x}^{-1 / 4} N u_{x}$ \\
\hline 0 & 0.7 & 0.40417 & 0.42914 \\
100 & 0.7 & 0.21340 & 0.31129 \\
200 & 0.7 & 0.18086 & 0.29735 \\
0 & 5.4 & 0.29034 & 0.63663 \\
100 & 5.4 & 0.19981 & 0.46721 \\
200 & 5.4 & 0.17379 & 0.41867 \\
0 & 7.0 & 0.27647 & 0.66971 \\
100 & 7.0 & 0.19668 & 0.50048 \\
200 & 7.0 & 0.17201 & 0.44725 \\
\hline
\end{tabular}

diffusivity for a fixed kinematic viscosity $v$, it opposes conduction. This results in thinner thermal and momentum boundary layers. Hence, both temperature and velocity are reduced as $\operatorname{Pr}$ increases.

Table 5 gives the effect of the inertia parameter $C$ on the local skin friction coefficient and the Nusselt number $\left(2 G r_{x}^{1 / 4} C_{\mathrm{f} x}, G r_{x}^{-1 / 4} N u_{x}\right)$ for $S=0.5, P r=0.7,5.4,7.0$, $K=1, \varepsilon=0.9, \xi=2$. For a given Prandtl number, both the skin friction and the Nusselt number decrease with increasing $C$, because momentum and thermal boundary layers become thick due to the increase in the resistance to the motion.

The effect of the permeability parameter $K$ on the skin friction and the Nusselt number $\left(2 G r_{x}^{1 / 4} C_{\mathrm{f} x}, G r_{x}^{-1 / 4}\right.$ $N u_{x}$ ) for $S=0.5, P r=0.7,5.4,7.0, C=200, \varepsilon=0.9, \xi=2$ is presented in Table 6. For fixed $P r$, both the skin friction coefficient and the Nusselt number increase with $K$ due to the reduction in the resistance to the flow as mentioned earlier. 
Table 6

Local skin friction coefficient $\left(2 G r_{x}^{1 / 4} C_{\mathrm{f} x}\right)$ and local Nusselt number $\left(G r_{x}^{-1 / 4} N u_{x}\right)$ for $C=200, S=0.5, \varepsilon=0.9$ and $\xi=2.0$

\begin{tabular}{llcl}
\hline \multicolumn{1}{c}{$K$} & $P r$ & $2 G r_{x}^{1 / 4} C_{\mathrm{f} x}$ & $G r_{x}^{-1 / 4} N u_{x}$ \\
\hline 0.316 & 0.7 & 0.16665 & 0.28966 \\
1.0 & 0.7 & 0.18086 & 0.29735 \\
0.316 & 5.4 & 0.16080 & 0.39309 \\
1.0 & 5.4 & 0.17379 & 0.41867 \\
0.316 & 7.0 & 0.15927 & 0.41835 \\
1.0 & 7.0 & 0.17201 & 0.44725 \\
\hline
\end{tabular}

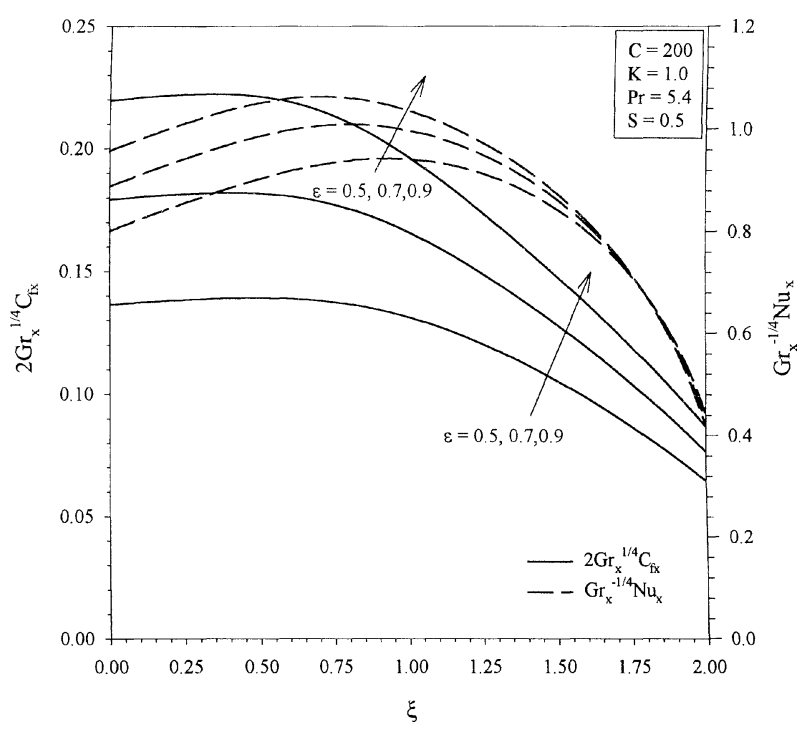

Fig. 13. Effect of porosity $\varepsilon$ on the local skin friction coefficient and the local Nusselt number $\left(2 G r_{x}^{1 / 4} C_{\mathrm{f} x}, G r_{x}^{-1 / 4} N u_{x}\right)$.

The effect of the porosity $\varepsilon$ on the local skin friction and the local Nusselt number $\left(2 G r_{x}^{1 / 4} C_{\mathrm{f} x}, G r_{x}^{-1 / 4} N u_{x}\right)$ for $S=0.5, P r=5.4, K=1, C=200,0 \leqslant \xi \leqslant 2$ is presented in Fig. 13. The effect of $\varepsilon$ is more pronounced for smaller values of $\xi(\xi<1)$. The reason for this trend is that the effect of other parameters, namely $K, C, S$ is comparatively small for $\xi<1$, since these parameters are multiplied by $\xi$. The effect of these parameters becomes significant for $\xi>1$ and they tend to reduce the effect of $\varepsilon$. Also for a fixed value of $\varepsilon$, the skin friction and the Nusselt number increase with $\varepsilon$, because the resistance to the flow decreases with increasing $\varepsilon$. This results in thinner momentum and thermal boundary layers. Consequently the velocity and temperature gradients and hence the skin friction and the Nusselt number are increased.

Fig. 14 represents the effect of the permeability $K$ on the local skin friction and the local Nusselt number $\left(2 G r_{x}^{1 / 4} C_{\mathrm{f} x}, G r_{x}^{-1 / 4} N u_{x}\right)$ for $S=0.5, P r=5.4, K=1$, $C=200, \varepsilon=0.9$, in the range $0 \leqslant \xi \leqslant 2$. Both the skin friction and the Nuselt number increase with increasing $K$. Consequently the momentum and thermal boundary layers are reduced. This results in higher skin friction and heat

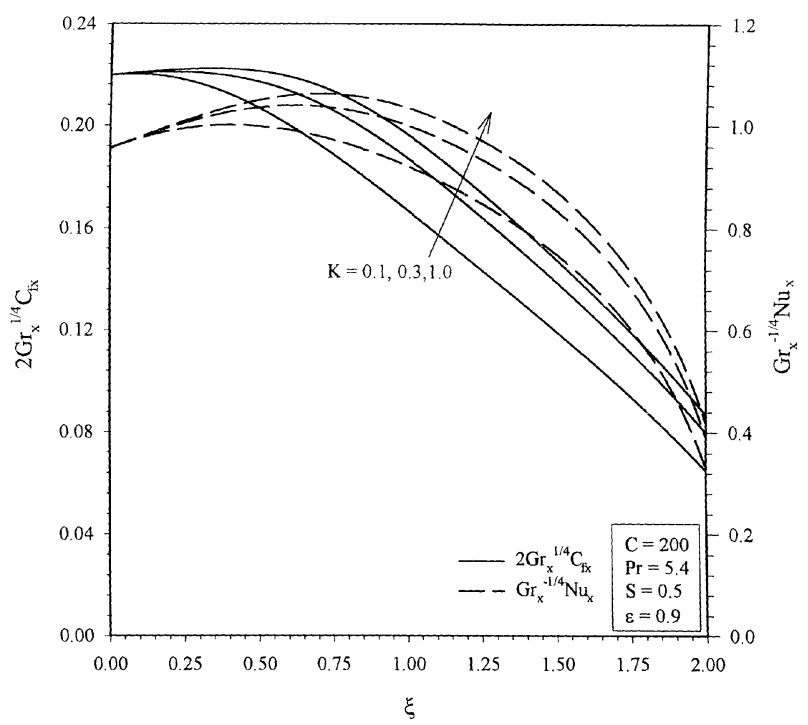

Fig. 14. Effect of permeability $K$ on the local local skin friction coefficient and the local Nusselt number $\left(2 G r_{x}^{1 / 4} C_{\mathrm{f} x}, G r_{x}^{-1 / 4} N u_{x}\right)$.

transfer for increasing permeability. Since $K$ is multiplied by $\xi$ (see Eq. (6)) its effect at $\xi=0$ vanishes.

\section{Conclusions}

The effect of the thermal stratification parameter on the local skin friction coefficient and the Nusselt number is more pronounced for moderate and large values of the curvature parameter $(\xi \geqslant 1)$. For certain values of the thermal stratification parameter, the wall of the cylinder gets heated instead of being cooled (i.e., the direction of the heat transfer changes). The skin friction coefficient vanishes for certain values of the stratification parameter or the curvature parameter. For small or moderate values of the curvature parameter, the results obtained by employing the perturbation method with Shanks transformation are found to be in very good agreement with those obtained by using the finite-difference method.

\section{References}

[1] C.L. Tien, K. Vafai, Convective and radiative heat transfer in porous media, Adv. Appl. Mech. 27 (1990) 225-281.

[2] C.K. Chen, C.I. Hung, H.C. Horng, Transient natural convection on a vertical flat plate embedded in a high porosity medium, ASME J. Energy Res. Tech. 109 (1987) 112-118.

[3] P. Singh, K. Tewari, Non-Darcy free convection from vertical surfaces in thermally stratified porous media, Internat. J. Engrg. Sci. 31 (1993) 1233-1242.

[4] C.K. Chen, C.R. Lin, Natural convection from an isothermal vertical surface embedded in a thermally-stratified high porosity medium, Internat. J. Engrg. Sci. 33 (1995) 131-138.

[5] A.J. Chamkha, Hydromagnetic natural convection from an isothermal inclined surface adjacent to a thermally stratified porous medium, Internat. J. Engrg. Sci. 35 (1997) 975-996.

[6] R. Eichhorn, Prog. Natural convection in a thermally stratified fluid, Heat Mass Transfer 2 (1969) 41-53. 
[7] C.C. Chen, R. Eichhorn, Natural convection from a vertical surface to a thermally stratified fluid, ASME J. Heat Transfer 98 (1976) 446-451.

[8] B.J. Venkatachala, G. Nath, Non-similar natural convection in a thermally stratified fluid, Internat. J. Heat Mass Transfer 24 (1981) 18481850 .

[9] A.K. Kulkarni, H.R. Jacobs, J.J. Hwang, Similarity solution for natural convection flow over an isothermal wall immersed in thermally stratified medium, Internat. J. Heat Mass Transfer 30 (1987) 681-688.

[10] D. Angirasa, J. Srinivasan, Natural convection flows due to the combined buoyancy of heat and mass transfer in a thermally stratified medium, ASME J. Heat Transfer 111 (1989) 657-663.

[11] C.C. Chen, R. Eichhorn, Natural convection from spheres and cylinders immersed in a thermally stratified fluid, ASME J. Heat Transfer 101 (1979) 566-569.

[12] F.G. Blottner, Finite difference methods of solution of boundary layer equations, AIAA J. 8 (1970) 193-205.

[13] A. Aziz, T.Y. Na, Improved perturbation solutions for laminar natural convection on a cylinder, Warme-und Stoffubertragung 83 (1982) 83 87.
[14] E.J. Lefevre, A.J. Ede, Laminar free convection from the outer surface of a vertical circular cylinder, in: Proc. Conf. Appl. Mech., Vol. 4, 1956, pp. 175-183.

[15] E.R. Hema, J.V. Recesso, J. Christiaens, The axisymmetric free convection temperature field along a vertical thin cylinder, J. Aerospace Sci. 26 (1959) 335-342.

[16] T. Cebeci, Laminar free convection heat transfer from the outer surface of a vertical cylinder, Proc. Internat. Heat Transfer Conf. N.C. 14 (1974) 15-19.

[17] W.J. Minkowycz, E.M. Sparrow, Local non-similar solution for natural convection on a cylinder, ASME J. Heat Transfer 96 (1974) 178-183.

[18] D. Shanks, Non-linear transformations of divergent and slowly convergent sequences, J. Math. Phys. 34 (1955) 1-42.

[19] T.Y. Na, Computational Methods in Engineering Boundary Value Problems, Academic Press, New York, 1979. 